\title{
Early treatment of acute promyelocytic leukaemia is accurately guided by the PML protein localisation pattern: real-life experience from a tertiary New Zealand centre
}

\author{
${\text { Nicole } \text { Chien }^{1} \text {, Michelle Petrasich }}^{2}$, George Chan ${ }^{1,2}$, Edward Theakston ${ }^{2}$, \\ Anna Ruskova ${ }^{2}$, Nicola Eaddy ${ }^{1,2}$, Timothy Hawkins ${ }^{1}$, Leanne Berkahn ${ }^{1}$, \\ Richard Doocey $^{1}$, Peter J. Browett ${ }^{1,2,3}$, Taryn N. Green ${ }^{3}$, \\ MAGgie L. KALEV-ZYLINSKA ${ }^{2,3}$ \\ ${ }^{1}$ Department of Haematology, Auckland City Hospital, Auckland, New Zealand; ${ }^{2}$ Department \\ of Pathology and Laboratory Medicine, LabPlus, Auckland City Hospital, Auckland, New \\ Zealand; ${ }^{3}$ Department of Molecular Medicine and Pathology, School of Medical Sciences, \\ University of Auckland, Auckland, New Zealand
}

\begin{abstract}
Summary
Current guidelines recommend that a rapid test be used to assist diagnosis of acute promyelocytic leukaemia (APL), but the choice of an assay is discretionary. PML immunofluorescence (PML IF) identifies the microparticulate pattern of the PML protein localisation, highly specific for APL. The aim of this study was to evaluate clinical utility of PML IF in a real-life setting based on a retrospective records review for all patients who had PML IF performed in our centre between 2000 and 2017.

Final analysis included 151 patients, 70 of whom had APL. PML IF was reported on average 3 days faster than cytogenetics. Compared with genetic results, PML IF showed sensitivity of $96 \%$ and specificity of $100 \%$. PML IF accurately predicted APL in four APL cases with cryptic karyotype/FISH and excluded APL in $98 \%$ cases tested based on the suspicious immunophenotype alone, 21/28 of whom had mutated NPM1. Results of PML IF influenced decision to start ATRA in 25 (36\%) APL patients and led to its termination in six non-APL patients.

In conclusion, PML IF is a fast and reliable test that facilitates accurate treatment decisions when APL is suspected. This performance of PML IF remains hard to match in a real-life setting.
\end{abstract}

Key words: PML immunofluorescence; PML protein localisation; promyelocytic leukaemia protein; rapid diagnosis; rapid treatment.

Received 31 October, revised 19 December 2018, accepted 5 January 2019 Available online 12 March 2019

\section{INTRODUCTION}

The ProMyelocyticLeukaemia (PML) protein, a critical organiser of nuclear bodies, is encoded by the $P M L$ gene located on chromosome $15 \mathrm{q} 22 .^{1,2} P M L$ is rearranged in almost all cases of acute promyelocytic leukaemia (APL) through $\mathrm{t}(15 ; 17)(\mathrm{q} 22 ; \mathrm{q} 11-12)$ chromosomal translocation that fuses $P M L$ with the Retinoic Acid Receptor Alpha (RARA) gene. $^{3}$ APL patients often present with significant coagulopathy and life threatening haemorrhages, hence urgent diagnosis is required to initiate treatment with all-transretinoic acid (ATRA). ${ }^{4,5}$ APL is often suspected based on the characteristic morphology of abnormal promyelocytes (coarse granules, multiple Auer rods or bilobed nuclei) and their distinctive immunophenotype (lack of expression of CD34 and HLA-DR). ${ }^{6}$ However, the hypogranular APL variant can be more difficult to recognise, and loss of expression of CD34 and HLA-DR also occur in other types of acute myeloid leukaemia (AML). ${ }^{7}$ Definitive diagnosis of APL requires molecular confirmation of PML-RARA using cytogenetics, fluorescence in situ hybridisation (FISH) or reverse transcription polymerase chain reaction (RT-PCR). ${ }^{6}$ Because genetic methods have relatively long turn-around times some centres, including ours, adopted a rapid and simple PML immunofluorescence test (PML IF) that can strengthen provisional diagnosis of APL within hours. ${ }^{8,9}$

The principle of PML IF is based on the observation that in normal cells, PML assembles into distinct nuclear bodies, whereas in the presence of PML-RARA, PML assembly is disrupted. ${ }^{10,11}$ PML IF visualises scattered nuclear localisation of the PML protein, which is a highly sensitive and specific feature of APL, contributing to its pathogenesis. ${ }^{12}$ PML IF can be normal in rare variants of APL that carry rearrangements of genes other than $P M L$, such as NPMI (Nucleophosmin 1), NUMA1 (Nuclear Mitotic Apparatus 1), STAT5B (Signal Transducer and Activator of Transcription $5 B$ ) and ZBTB16 (Zinc Finger and BTB Domain Containing 16), previously called PLZF (Promyelocytic Leukemia Zinc Finger). However, because these variants are less responsive to ATRA, normal PML IF is considered not to disadvantage these patients in the acute setting. ${ }^{13,14}$

Our hospital is a tertiary public hospital and a clinical research facility that provides specialist leukaemia care to approximately 1.7 million people. We introduced PML IF to assist rapid diagnosis of APL in the year 2000. The test is performed on request from a haematologist when APL is suspected based on either morphology, immunophenotype or coagulopathy. The perception has developed in our group that the assay has been very useful but its clinical utility has never 
been formally evaluated. This study reviewed the use of PML IF in our centre since its introduction, with an overriding aim to help guide further improvements. We examined features that triggered requests for PML IF and correlated results with other morphological, immunophenotypical and molecular findings. In addition, we compared real-life reporting times for different assays, together with the impact of results on the decision to initiate or terminate ATRA. Our findings emphasise the clinical usefulness of PML IF and reveal that clinicians veer on the side of confidence in their decisions to start ATRA. In the Discussion, we reflect on alternative strategies that could be used to assist rapid diagnosis of APL.

\section{MATERIALS AND METHODS}

\section{Study design}

This was a retrospective review of clinical and laboratory records for all patients (paediatric and adults) who had the PML IF performed in our institution between February 2000 and January 2017. Cross-reference was made with the molecular results database to ensure all APL patients were captured. Comprehensive clinical data were recorded, including demographics, presenting full blood count, peripheral blood morphology, baseline coagulation results, morphological diagnosis on the bone marrow aspirate, blast immunophenotype, karyotype, FISH and RT-PCR for PML-RARA, together with the reporting times of the diagnostic assays and the time to start ATRA. All study procedures were approved by the institutional research review committee (approval number $\mathrm{A}+7753)$.

\section{PML immunofluorescence test}

Our PML IF method was developed based on the indirect staining procedure previously described. ${ }^{11,15}$ Briefly, cytospin or smear preparations of peripheral blood or marrow cells were well air-dried and fixed in 1:1 methanol:acetone mixture at $-20^{\circ} \mathrm{C}$ for $90 \mathrm{~s}$. Mouse monoclonal antihuman PML antibody (PG-M3; Santa Cruz Biotechnology, USA) was diluted 1:25 in phosphate buffered saline (PBS; containing $2 \%$ foetal calf serum and $0.01 \%$ sodium azide) and incubated with cells for $30 \mathrm{~min}$ at room temperature (RT). Slides were washed in a cold PBS bath for $5 \mathrm{~min}$, then incubated at RT for $1 \mathrm{~h}$ with a secondary, sheep anti-mouse, fluorescein isothiocyanate (FITC) labelled immunoglobin G (Sigma-Aldrich, USA) diluted 1:100 as above. Slides were washed as above, mounted using Fluoprep (bioMerieux, France) and examined under an Olympus BH2 fluorescent microscope with a $40 \times$ oil lens and phase. Positive and negative controls of frozen samples (previous APL and other AML, respectively) were included in each testing round. Typical turn-around time of the test is $4 \mathrm{~h}$. Results are phoned through to the requesting physician when the assay has been completed.

\section{Cytogenetics, FISH and RT-PCR for PML-RARA}

Molecular tests were performed according to standard protocols, as described before. $^{16}$

\section{Statistical analysis}

Data were analysed using SPSS Statistics software and are shown as mean, median, standard error of the mean (SEM) and standard deviation (SD), as indicated. Proportions between groups were examined using Pearson $\chi^{2}$ test if $n>5$ or Fisher exact if $n<5$. Mean differences between groups were analysed using independent-samples $t$-test (two-sided) or one-way analysis of variance (ANOVA). Pearson correlation and linear regression were applied to test the relationship between two scalar variables. $p$ values less than 0.05 were considered statistically significant.

\section{RESULTS}

We identified 182 patients who had PML IF performed at the time of leukaemia diagnosis between February 2000 and January 2017; 31 patients were excluded from further evaluation because of the lack of adequate clinical data, leaving
151 for the analysis. The total number of PML IF performed in each year ranged from 1 to 18, median 9, SD 4 (Fig. 1A). Samples where leukaemic cells contained up to 20 distinct nuclear bodies were reported as normal (the wild-type pattern, negative result) and those with dispersed nuclear appearances as consistent with APL (the microparticulate pattern, positive result; Fig. 1B). ${ }^{11}$ The wild-type pattern was reported in $82(54 \%)$ patients, microparticulate in $67(45 \%)$ and only two results $(1 \%)$ were indeterminate. The ratio between negative and positive results remained relatively constant over the years, indicating consistent demand for PML IF to both rule out and confirm APL (Fig. 1A). When crossreferenced against the molecular database, we found that all but one APL patient diagnosed during the study period had PML IF performed.

Most PML IF $(108 ; 72 \%)$ were carried out on bone marrow samples, $38(25 \%)$ on peripheral blood and five (3\%) on both blood and marrow. PML IF was conducted after hours for 53 patients, including eight during weekends. Difficulties in the conduct or reporting of the test were documented for nine $(6 \%)$ tests. Seven of these tests were repeated and a conclusive result was reached in five. The main reason for the difficulties was the lack of intact cells on the slides, encountered for six patients. Three of these patients received ATRA prior to testing, which was thought to be the cause. Consequently in 2003, the lab recommended that the test be performed on samples collected prior to the start of ATRA, if possible. The staining pattern itself was difficult to interpret for four patients. In two, the result was established upon review by senior staff. The result remained indeterminate for two patients, primarily due to the lack of intact cells.

\section{Triggers for requesting PML immunofluorescence}

Morphology was identified as a sole trigger for testing in $14(9 \%)$ patients, morphology and immunophenotype in $71(47 \%)$ and immunophenotype alone in 54 (36\%) (Supplementary Table 1, Appendix A). Coagulopathy was recorded in 42 patients but it was a sole trigger for testing in only one. In four patients, the reason for performing PML IF was not clear from the available documentation.

Morphology was a strong predictor of the positive PML IF. Of 85 patients suspected to have APL based on morphology, $67(79 \%)$ had a positive result. In contrast, suspicious immunophenotype by itself correlated poorly with APL. Of 54 patients tested solely on the basis of the abnormal immunophenotype, without supportive morphological characteristics, only one (2\%) returned a positive result.

\section{Correlations with genetic findings}

Of 67 cases with a microparticulate pattern of PML IF, all were confirmed to have $P M L-R A R A$ present using cytogenetics, FISH or RT-PCR (Table 1), meaning PML IF generated no false positive results. On the other hand, of 82 cases with the wild-type pattern, only one was found to have PML-RARA, meaning PML IF generated one false negative result. The two indeterminate cases were found to be $P M L-R A R A$ positive, meaning PML IF missed two APL cases (Table 1). Overall, PML IF had a sensitivity of $96 \%$ and specificity of $100 \%$ in our cohort of 151 patients 
A

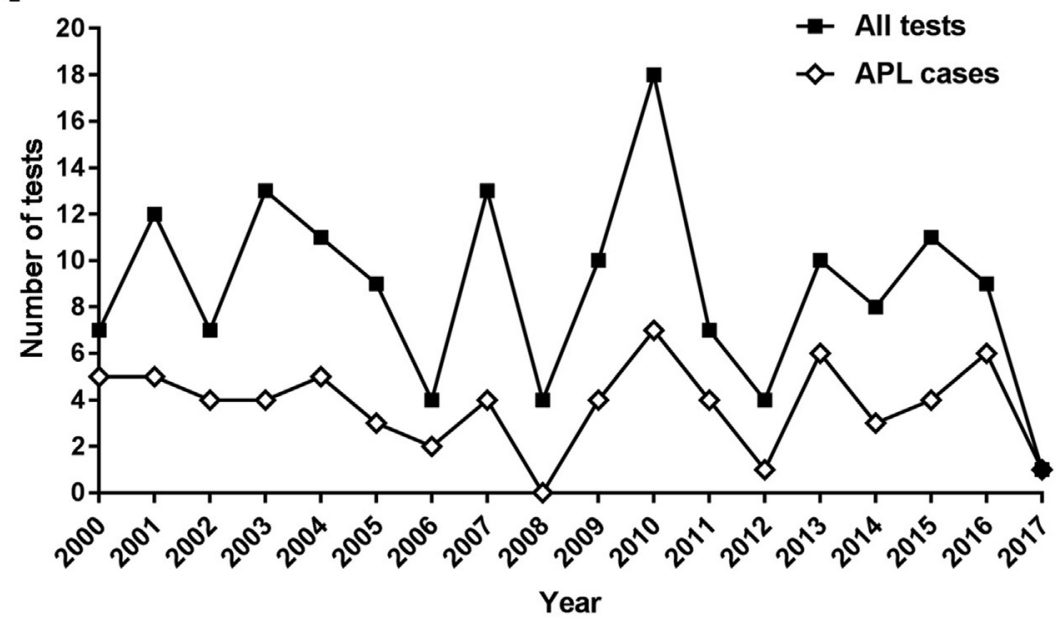

B

i

ii
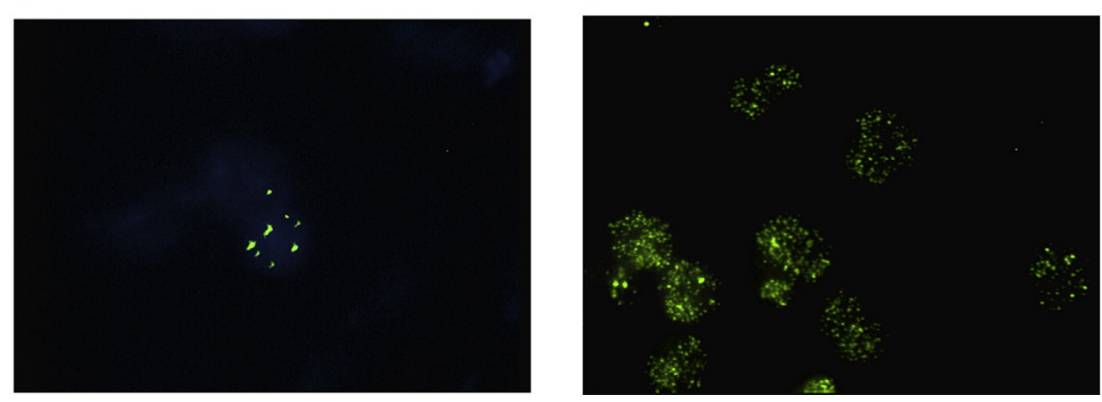

Fig. 1 PML immunofluorescence - test numbers and interpretation. (A) Number of PML immunofluorescence (PML IF) tests performed in our centre between February 2000 and January 2017; total and positive tests are shown per year. (B) Distinct patterns of PML IF used as a predictor of PML-RARA. (B.i) The wild-type, normal pattern of PML localisation seen within the nucleus of non-APL cell; this image had the contrast enhanced using Cytovision. The PML protein localises to distinct nuclear bodies (pods, typically 5-10 per cell) visualised as discrete dots that can be easily counted. The percentage of such cells in non-APL samples varied between leukaemia types but was typically $<50 \%$. (B.ii) The microparticulate pattern of PML localisation within the nucleus of APL cells. Staining is dispersed with scattered fine dots that are difficult to count (typically $>30$ per cell). Barring problems with cell preservation, APL samples typically contained $>95 \%$ of positive cells.

Table 1 Summary of diagnostic results for patients suspected to have APL according to the final diagnosis

\begin{tabular}{lll}
\hline & APL, $n(\%)$ & non-APL, $n(\%)$ \\
\hline Total number & 70 & 81 \\
Type of sample used for PML IF & & \\
Peripheral blood & $15(21 \%)$ & $23(28 \%)$ \\
Bone marrow & $51(73 \%)$ & $57(71 \%)$ \\
Both & $4(6 \%)$ & $1(1 \%)$ \\
PML IF result & $67(96 \%)$ & 0 \\
Microparticulate pattern & $1(1 \%)$ & $81(100 \%)$ \\
Wild-type pattern & $2(3 \%)$ & 0 \\
Indeterminate & $46(66 \%)$ & 0 \\
Karyotype & $16(23 \%)$ & 0 \\
t(15;17) & $3(4 \%)$ & $46(57 \%)$ \\
t(15;17) with secondary changes & $2(3 \%)$ & $26(32 \%)$ \\
Normal & $3(4 \%)$ & $9(11 \%)$ \\
Other abnormalities & & \\
Not done & $48(69 \%)$ & 0 \\
FISH & $3(4 \%)$ & $24(30 \%)$ \\
$P M L-R A R A$ gene fusion detected & $19(27 \%)$ & $57(70 \%)$ \\
$P M L-R A R A$ gene fusion not detected & $51(73 \%)$ & 0 \\
Not done or not available & 0 & $8(10 \%)$ \\
RT-PCR & $19(27 \%)$ & $73(90 \%)$ \\
PML-RARA transcripts detected & & \\
$P M L-R A R A$ transcripts not detected & & \\
Not done or not available & & \\
\hline
\end{tabular}


comprised of 70 APL and 81 non-APL cases verified by molecular testing.

We took a closer look at the cases with discordant or uncertain results. PML IF accurately predicted APL in four patients with a negative karyotype, including two in whom FISH was negative (Table 2). Regarding the false negative PML IF case, there were no circulating blasts but marrow morphology was highly suspicious for APL. The slides on which PML IF was performed had very few cells, some of which had a microparticulate pattern but the majority of cells appeared to have the wild-type pattern, hence the negative result was issued. This patient had cryptic PML-RARA gene rearrangement on karyotype and FISH, and APL was eventually confirmed by RT-PCR (Table 2).

Amongst non-APL cases, there were no false positive PML IF results. Nevertheless, there was one case where the result was initially indeterminate and required review by senior staff, leading to some initial confusion and a delay in reporting.

\section{Comparison of test reporting times}

Because we could not reliably establish exact times when different assays were requested for all patients, we expressed times to formal result reporting relative to the time of admission. This clearly overestimates true turn-around times, but still provides a relative measure of the speed of various testing. PML IF reports were available the fastest, on day 1.65 \pm 1.7 after admission (Fig. 2, Table 3). Molecular results were reported later, cytogenetics on day $4.95 \pm 3.2$, FISH on day $5.06 \pm 5.1$ and RT-PCR on day $21.89 \pm 12.3$ after admission $(p<0.0001$ for all compared with the reporting time of PML IF). For all assays, positive results were reported faster than negative results, reflecting effective test prioritisation in the laboratory (Fig. 2, Table 3).

\section{Impact of PML immunofluorescence results on ATRA treatment}

ATRA was started before PML IF was reported in 39 patients; this included 32 confirmed APL and seven non-APL patients (Fig. 3). Most (35) of these patients had morphological features of APL either in the peripheral blood or bone marrow.
The remaining four patients were started on ATRA due to coagulopathy and/or suspicious immunophenotype and all these were found not to have APL. Nevertheless, of 57 patients suspected to have APL from peripheral blood morphology (seven of whom were found not to have APL), only $33(58 \%)$ were commenced on ATRA prior to issuing a PML IF report, suggesting clinicians often waited for the PML IF result before starting ATRA. PML IF results were identified as a trigger for starting ATRA in 25 APL patients. Three APL patients who had indeterminate $(n=2)$ or wild-type $(n=1)$ PML IF started ATRA based on morphology. Four patients commenced ATRA based only on molecular results, despite the PML IF report being available earlier. The median time to starting ATRA in APL patients was $11.8 \mathrm{~h}$ (range 0-104 h) from admission; $50 \%$ patients started ATRA on the day of admission, $24 \%$ within $48 \mathrm{~h}$ and $10 \%$ within $72 \mathrm{~h}$.

In the non-APL group, seven patients were put on ATRA prior to the PML IF result based mostly on morphology or abnormal coagulation (Fig. 3). Six of these patients had ATRA stopped after the negative PML IF result was issued. One patient who continued ATRA despite the normal PML IF had significant coagulopathy and lacked HLA-DR and CD34 expression, which raised concerns of the APL variant. Another non-APL patient commenced on ATRA despite a normal PML IF result, driven by morphological and coagulation abnormalities. Ultimately, RT-PCR was negative in both of these patients and ATRA was discontinued.

Overall, 25/70 (36\%) APL patients, and 31/151 (21\%) of the entire cohort had ATRA therapy modified based on the informative (positive or negative) PML IF result. The facilitatory impact of PML IF on treatment decisions is also reflected by a moderate correlation we found between the times taken to report PML IF and to start ATRA $(p=0.002$; Fig. 4A). This correlation was weaker for cytogenetics ( $p=0.071$; Fig. 4B), implying that by the time cytogenetics were reported, most patients were already on ATRA.

\section{Laboratory characteristics of APL and non-APL patients}

Various laboratory characteristics were reviewed from the time of admission with an aim to seek features predictive of

Table 2 Summary of discrepant and uncertain diagnostic results in patients suspected to have APL highlighting contribution of PML immunofluorescence

\begin{tabular}{|c|c|c|c|c|}
\hline Case no. & Karyotype & FISH for $P M L-R A R A$ & RT-PCR for $P M L-R A R A$ & PML IF \\
\hline \multicolumn{5}{|c|}{ Discrepant cases } \\
\hline 41 & Normal & $\mathrm{Neg}$ & Pos & $\operatorname{Pos}^{\mathrm{a}}$ \\
\hline $69^{\mathrm{b}}$ & $46, X Y, \operatorname{del}(14)(q 11 q 21), ? t(15 ; 17)(q 22 ; p 11)[20]$ & Pos & Pos & $\operatorname{Pos}^{\mathrm{a}}$ \\
\hline $76^{\mathrm{b}}$ & $\begin{array}{l}46, \text { XY,der(15)(15pter->15q1?2::17p11?2->17pter),der(17)(17qter- } \\
\quad>17 \mathrm{q} 21:: 15 \mathrm{q} 22->15 \mathrm{q} 1 ? 2:: 17 \mathrm{p} 11 ? 2->17 \mathrm{q} 21:: 15 \mathrm{q} 22->15 \mathrm{qter})[20]\end{array}$ & Pos & Pos & $\operatorname{Pos}^{\mathrm{a}}$ \\
\hline \multicolumn{5}{|c|}{ Uncertain cases } \\
\hline 154 & ND & ND & ND & $\operatorname{Pos}^{\mathrm{d}}$ \\
\hline 127 & ND & Pos & ND & $\operatorname{Pos}^{\mathrm{d}}$ \\
\hline 74 & ND & Pos & Pos & $\operatorname{Pos}^{\mathrm{d}}$ \\
\hline
\end{tabular}

APL, acute promyelocytic leukaemia; FISH, fluorescence in situ hybridisation; IF, immunofluorescence; ND, not done or failed; Neg, negative; Pos, positive; RTPCR, reverse transcription polymerase chain reaction.

${ }^{a}$ PML IF predicted cytogenetically cryptic PML-RARA in four patients, including two with negative FISH.

${ }^{\mathrm{b}}$ Cases 69 and 76 had no $\mathrm{t}(15 ; 17)(\mathrm{q} 22 ; \mathrm{q} 11-12)$ reported on the karyotype.

${ }^{c}$ This was a false negative result in a case with cryptic cytogenetics and FISH.

${ }^{\mathrm{d}}$ Three patients had no karyotype obtained, and positive PML IF triggered further molecular assays for PML-RARA. 
A

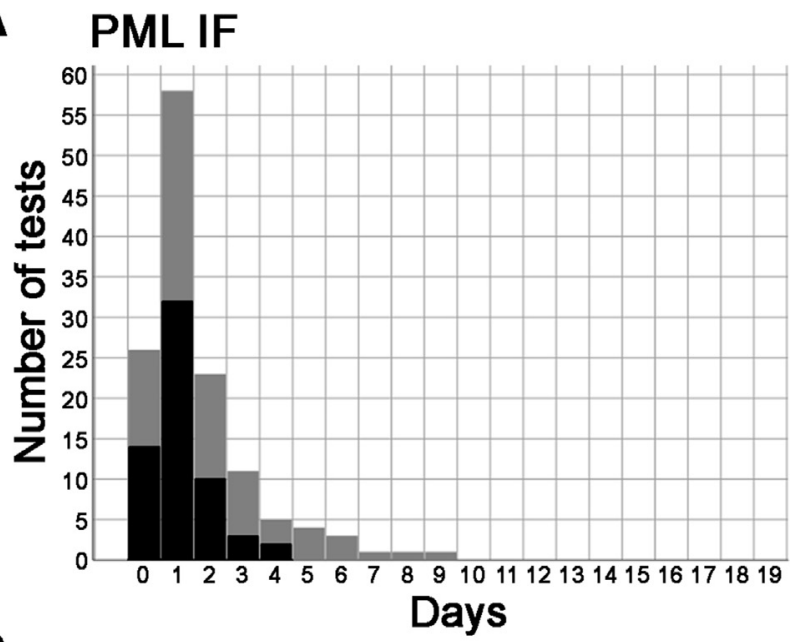

B

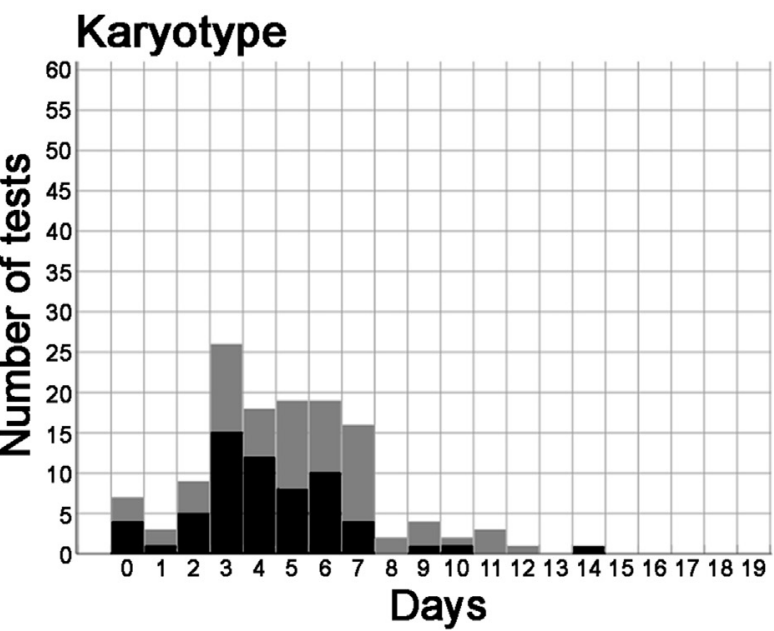

C

FISH

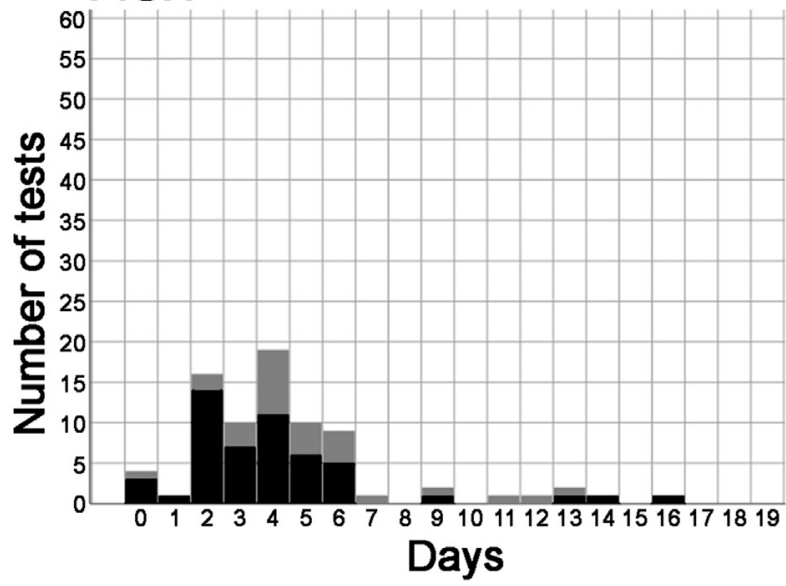

- APL $\square$ non-APL

Fig. 2 Comparison of reporting times for selected assays used to confirm APL diagnosis. Stacked bars indicate numbers of tests formally reported on consecutive days counted from the day of admission (day 0) until day 19 for PML immunofluorescence (PML IF; A), karyotype (B) and FISH for PMLRARA (C) graphed separately for patients with APL (black bars) and non-APL patients (grey bars). Some patients had their tests requested before admission, contributing to the availability of results on day 0 . Times taken to obtain karyotype and FISH results were significantly longer than for PML IF. Most RTPCR tests were reported beyond this time-frame, hence are not shown. Statistical analysis for all test reports, including those reported beyond day 19, and the RT-PCR data are shown in Table 3.
APL (Table 4). APL patients presented with lower platelet and white cells counts, including lower counts of blasts/blast equivalents and neutrophils, compared with non-APL patients. Fibrinogen levels were also lower in APL patients, albeit still within the normal range; activated partial thromboplastin time (APTT) and prothrombin ratio (PR) were not prolonged.

Flow cytometry immunophenotyping was performed on bone marrow samples in 113 patients, peripheral blood in 26, and both blood and marrow in 11 patients. Mean expression of HLA-DR and CD34 was less than $20 \%$ in this study, as this comprised a trigger for PML testing. Nevertheless, confirmed APL cases still had lower expression of HLA-DR, as well as CD117, CD56 and CD11c, compared with nonAPL cases (Table 4). In comparison, expression of CD13 and CD64 was higher but the actual difference appeared too small to be diagnostically useful (Table $4, p<0.05$ for all).

In small numbers of confirmed APL cases, the blast immunophenotype was atypical with stronger expression of CD34 (detected on up to $82 \%$ blasts) and HLA-DR (up to $50 \%$ ), emphasising that APL cannot be excluded based on maintained expression of CD34 and HLA-DR (Supplementary Table 2, Appendix A).

For patients who had both blood and marrow samples phenotyped, we checked if there was a discrepancy in the expression of CD34 and HLA-DR on blasts derived from the peripheral blood and marrow, but this was not found (Supplementary Table 3, Appendix A).

Morphological diagnosis of non-APL cases that initially mimicked APL, mostly by the immunophenotype, consisted mainly of AML without maturation (FAB M1;37\%) or with maturation (FAB M2; 27\%); the latter included five $(6 \%)$ patients with $\mathrm{t}(8 ; 21)$ translocation. Forty-six $(30 \%)$ patients had normal karyotype. Others had various karyotypic changes with no clear cytogenetic subgroup. Since 2007, all patients with intermediate risk AML suitable for intensive induction chemotherapy have been tested in our lab for mutations in FMS-like Tyrosine Kinase 3 (FLT3) gene, and since 2009, in NPM1. Together, 28 patients in this cohort were tested for FLT3 and NPMI mutations with the following outcomes: 17 had FLT3-ITD (internal tandem duplication) mutations, 15 of these also had concurrent NPM1 mutations; five had NPM1 mutations only; one had FLT3-TKD (tyrosine kinase domain) and NPM1 mutations; three were negative for either mutation. Our findings emphasise that AML with mutated NPM1 often have mature blast immunophenotype, which can trigger concerns about APL.

\section{DISCUSSION}

This study examined the clinical utility of PML IF in the rapid diagnosis of APL in a single, tertiary centre over the period of 17 years. We analysed full laboratory and clinical data for 151 patients, including 70 with APL. PML IF showed high sensitivity and specificity (96\% and 100\%, respectively) with a significantly shorter reporting time compared to cytogenetics, FISH and RT-PCR. PML IF was particularly useful to indicate APL diagnosis in cases with cryptic karyotype or FISH, where RT-PCR took the longest to report. Our study emphasises that PML IF is not just a poor man's alternative to molecular testing but a valuable assay that facilitates rapid detection of APL in a real-life setting, in accordance with current guidelines. ${ }^{17-19}$ 
Table 3 Time in days to reporting of various diagnostic APL tests calculated from the time of admission according to the final diagnosis

\begin{tabular}{|c|c|c|c|c|}
\hline \multirow[t]{2}{*}{ Test } & Total & APL & non-APL & \multirow[t]{2}{*}{$p$ value } \\
\hline & Mean \pm SD (n) & Mean \pm SD (n) & Mean \pm SD (n) & \\
\hline PML IF & $1.65 \pm 1.675(133)$ & $1.13 \pm 0.939(61)$ & $2.08 \pm 2.012(72)$ & 0.001 \\
\hline Karyotype & $\begin{array}{c}4.95 \pm 3.186(131) \\
p<0.0001^{\mathrm{b}}\end{array}$ & $4.27 \pm 2.390(62)$ & $5.57 \pm 3.672(69)$ & 0.020 \\
\hline FISH for $P M L-R A R A$ & $\begin{array}{c}5.06 \pm 5.147(80) \\
p<0.0001^{\mathrm{b}}\end{array}$ & $4.35 \pm 3.824(52)$ & $6.39 \pm 6.860(28)$ & 0.090 \\
\hline RT-PCR for $P M L-R A R A$ & $\begin{array}{c}21.89 \pm 12.333(89) \\
p<0.0001^{\mathrm{b}}\end{array}$ & $18.65 \pm 8.290(49)$ & $25.85 \pm 15.145(40)$ & 0.006 \\
\hline
\end{tabular}

APL, acute promyelocytic leukaemia; FISH, fluorescence in situ hybridisation; IF, immunofluorescence; RT-PCR, reverse transcription polymerase chain reaction; $\mathrm{SD}$, standard deviation.

${ }^{\mathrm{a}} p$ values refer to the comparison of reporting times between APL and non-APL cases. Positive results were reported faster.

${ }^{\mathrm{b}} p$ values refer to the comparison of reporting times against the time to PML IF reporting. Karyotype, FISH and RT-PCR for $P M L-R A R A$ were reported later than PML IF.

The performance of PML IF in our hands is in line with other international centres. Previous studies involving 15-199 APL patients reported sensitivity of PML IF ranging from $93 \%$ to $99 \%$ and specificity from $93 \%$ to $100 \% .^{8,9,11,15,20-22}$ The largest series was from MD Anderson Cancer Center, with 349 patients tested between 1996 and 2008, including 199 APL, showing PML IF sensitivity and specificity of $99 \%$ for both. ${ }^{8}$ The turn-around time for PML IF was quoted as $<4 \mathrm{~h}$ in that study, and for RT-PCR and FISH usually $24 \mathrm{~h}$. The latter appear significantly shorter than in our centre, however our analysis was based on reporting times, not true turn-around times. The long time for RT-PCR reporting in our lab reflects mainly the work-flow strategy where these tests are not required for diagnosis, hence not processed urgently and non-APL cases are assigned a lower priority for testing.

Although our report is not unique, it is one of the largest. $8,9,22$ Further, we expand on previous papers by adding new information on the triggers for PML IF testing, how these correlate with test results and the impact of results on treatment decisions. We found that when the request for PML IF was based on morphology, $79 \%$ of patients had APL. Therefore, one could ask if PML IF is needed when morphology is diagnostic, and should these patients be started on ATRA sooner? Current guidelines recommend that patients should be started on ATRA as soon as APL is suggested based on peripheral blood morphology, ${ }^{18,23,24}$ which did not happen for 17/56 (30\%) patients in our cohort, six of whom turned out not to have APL. In contrast, low CD34 and HLA-DR expression without characteristic morphology, or coagulopathy alone, were poorly predictive of APL, suggesting that PML IF may not be needed for these cases, or a negative result may eliminate the need for FISH and RTPCR in this context.

Although our correlations with morphology are very strong, we need to consider important limitations. Laboratory haematologists often wait for PML IF results before finalising bone marrow aspirate reports; therefore, morphological conclusions in this study may be more confident than they would be if PML IF were not available. Our results suggest clinicians prefer more certainty before starting ATRA without risking a delay in induction chemotherapy or ATRA side-effects in non-APL patients. This approach can be criticised as delays in ATRA administration have been shown to contribute to early haemorrhagic death; ${ }^{25}$ however, our testing strategy did not cause any undue delays in starting ATRA compared with the above study. ${ }^{25}$ In fact, therapeutic decisions may have been faster, likely due to a higher diagnostic confidence. A number of other confounding factors should also be noted. Treatment decisions are very

72 patients treated with ATRA
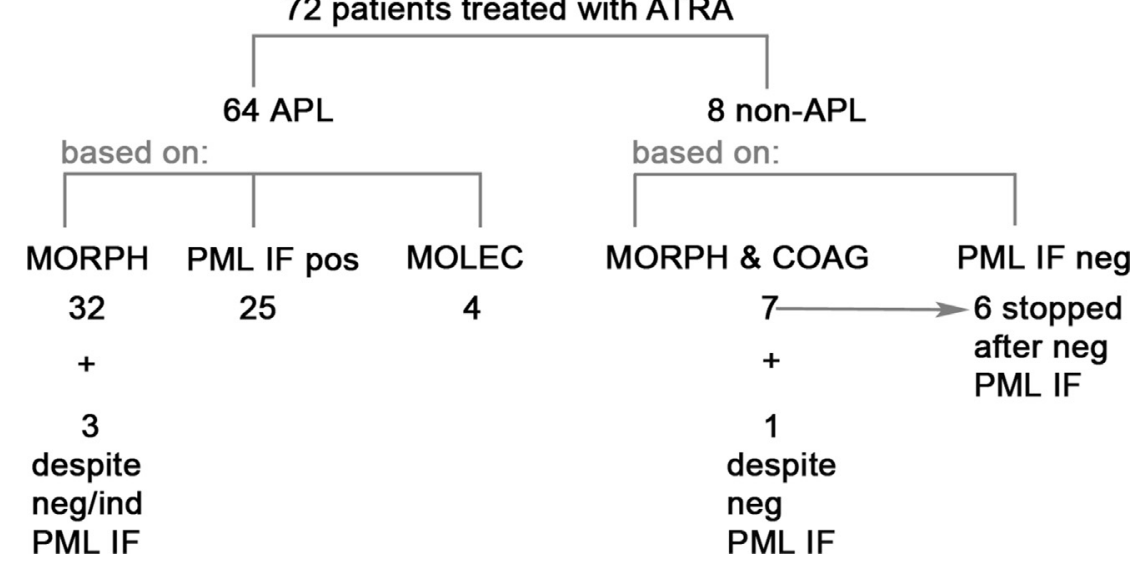

Fig. 3 Summary of triggers influencing decision to start and stop ATRA. APL, acute promyelocytic leukaemia; COAG, abnormal coagulation results; ind, indeterminate results; MOLEC, molecular test positive for PML-RARA; MORPH, morphology typical for APL; neg, negative result; PML IF, PML immunofluorescence; pos, positive result. 
A

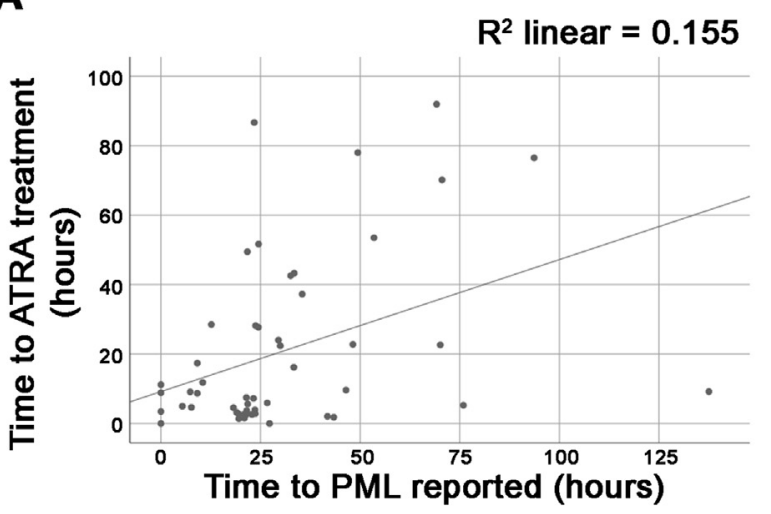

$$
\begin{aligned}
& \text { Pearson correlation }=0.393 \\
& P=0.002 \\
& \mathrm{n}=50
\end{aligned}
$$

B

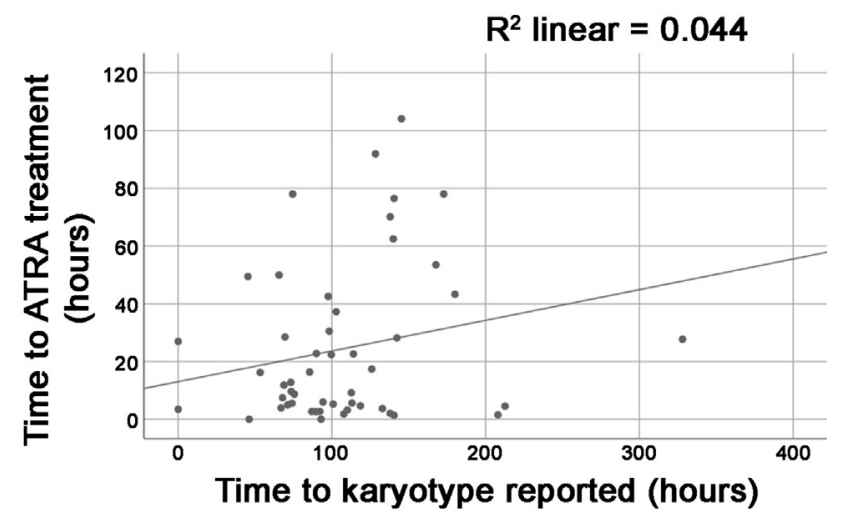

$$
\begin{aligned}
& \text { Pearson correlation }=0.211 \\
& P=0.071 \\
& \mathrm{n}=50
\end{aligned}
$$

Fig. 4 PML results influence the decision to start ATRA. Linear regression graphs, including lines of best fit with $\mathrm{R}^{2}$ values, Pearson $\mathrm{R}$ coefficient and significance values (1-tailed) are shown to demonstrate correlations between the times of starting ATRA and PML reporting (A) or karyotype reporting (B) analysed in hours from the time of admission until the formal release of the laboratory report. Some patients had their tests requested before admission, contributing to the availability of results at time 0 .

susceptible to individual clinician bias and despite thorough analysis of the timing of different laboratory reports and ATRA administration, we cannot be sure what laboratory information triggered a decision to start ATRA in individual patients. Nevertheless, our data argue that PML IF made bone marrow morphology reporting more conclusive in our centre, and strengthened clinical confidence in rapid treatment decisions. It was clear clinicians used PML IF results to both start and stop ATRA while molecular results were awaited.

International experience with PML IF remains limited, which is somewhat surprising, considering it is a simple assay. It appears the test is under-utilised where it could be most valuable. ${ }^{21,26}$ Should more be done to advocate PML IF utility to the wider world? In our centre, we would not dispose of it. Nevertheless, PML IF has its weaknesses. It is a manual test that adds workload in an increasingly busy and automated laboratory. Calling results requires experience that takes time to develop. Our staff are looking for an alternative but finding a suitable substitute is not straightforward.

A faster, direct (one-step) PML IF method could be used. Conjugates of anti-PML (PG-M3) antibodies are now commercially available (e.g., from Santa Cruz), providing a choice of fluorochromes, as well as peroxidase and alkaline phosphatase. In a study involving 30 patients with acute leukaemia, including nine APL patients, TRITC (tetramethylrhodamine-5-isothiocyanate) conjugated PG-M3 antibody was equally effective in determining PML localisation pattern as the indirect procedure we are currently using. ${ }^{27}$ Although immunocytochemistry is easier to examine under light microscopy and provides a permanent record, this method was less sensitive than indirect IF in earlier studies. ${ }^{15,20}$

Multi-parameter flow cytometry is unlikely to eliminate the need for PML IF but may reduce numbers of tests being required. The triple-negative cell marker profile of CD34, HLA-DR and CD11b identifies APL with sensitivity and specificity of approximately $93 \% .{ }^{28}$ Combined absence of CD11b, CD11c and HLA-DR may identify $96 \%$ APL. $^{29}$ In keeping with this, loss of CD11c correlated well with APL cases in our study. The addition of Class II-Associated Invariant Chain Peptide (CLIP) may also be helpful as CLIP is abundantly expressed on HLA-DR negative APL blasts but not on HLA-DR negative non-APL blasts. ${ }^{30}$ In another report, stepwise discriminant function analysis of seven antibodies targeting CD2, CD9, CD11b, CD13, CD34, CD117 and HLA-DR identified virtually all APL cases. $^{31}$

Unfortunately, conventional flow cytometry cannot resolve the pattern of PML protein localisation in leukaemic blasts. Specialised ImageStream (Luminex, USA) flow cytometry can visualise PML localisation in fixed and permeabilsed cells $^{32,33}$ but the equipment is expensive and not routinely available. Another modified flow cytometry approach was developed to detect PML-RARA fusion protein in cell lysates. ${ }^{34}$ Beads are coated with anti-RARA antibodies and applied to cell lysate to capture the fusion protein that is subsequently detected using PE (phycoerythrin) conjugated anti-PML antibody. A prototype of this test was promising when evaluated by EuroFlow Consortium ${ }^{34}$ but has not yet transitioned to routine diagnostics.

Finally, molecular methods are becoming easier and faster, and will likely offer the next best alternative. Rapid FISH $^{35}$ can achieve a result in $4 \mathrm{~h}$, which is similar to PML IF. Rapid digital PCR methods are also becoming available, ${ }^{36}$ as well as specialised biosensors capable of detecting $P M L$ RARA gene fusion. ${ }^{37}$

In conclusion, we have shown that PML IF is a useful test that assists rapid diagnosis and treatment of patients with APL in a tertiary hospital. We identified that PML IF based solely on lowish CD34 and HLA-DR expression seldom yields APL diagnosis, which may provide more confidence not to test if morphology is not supportive. Multi-parameter flow cytometry may reduce the number of PML tests being required, but the approaches proposed still require validation. In the longer-term, PML IF is likely to be superseded by a rapid and affordable molecular test. In the meantime, PML IF can be used to reliably assist rapid diagnosis and treatment of APL. 
Table 4 Comparison of baseline laboratory results for patients suspected to have APL according to the final diagnosis

\begin{tabular}{|c|c|c|c|}
\hline & APL & non-APL & $p$ value \\
\hline \multicolumn{4}{|l|}{ Full blood counts median, range (n) } \\
\hline Platelets $\left(\times 10^{9} / \mathrm{L}\right)$ & $26,5-204(67)$ & $67,5-467(80)$ & $<0.001$ \\
\hline White cell count $\left(\times 10^{9} / \mathrm{L}\right)$ & $2.4,0.2-166(68)$ & $12,0.3-471(80)$ & 0.001 \\
\hline Blasts/promyelocytes $\left(\times 10^{9} / \mathrm{L}\right)$ & $0.7,0-149(64)$ & $2.5,0-459(78)$ & 0.007 \\
\hline Neutrophils $\left(\times 10^{9} / \mathrm{L}\right)$ & $0.4,0-5.7(67)$ & $1.0,0-49.2(79)$ & 0.001 \\
\hline APTT & $31 \pm 5.2(68)$ & $33 \pm 6.9(75)$ & 0.023 \\
\hline $\mathrm{PR}$ & $1.5 \pm 1.6(68)$ & $1.2 \pm 0.3(75)$ & 0.126 \\
\hline Fibrinogen & $2.1 \pm 1.3(66)$ & $4.1 \pm 2.3(71)$ & $<0.001$ \\
\hline \multicolumn{4}{|c|}{ Cell markers, mean $\%$ positivity \pm SD $(n)$} \\
\hline HLA-DR & $8 \pm 17(66)$ & $19 \pm 32(73)$ & 0.02 \\
\hline CD34 & $14 \pm 23(66)$ & $14 \pm 30(74)$ & 0.932 \\
\hline CD117 & $46 \pm 30(57)$ & $71 \pm 30(69)$ & $<0.001$ \\
\hline CD13 & $84 \pm 22(64)$ & $73 \pm 33(71)$ & 0.022 \\
\hline CD33 & $94 \pm 16(64)$ & $93 \pm 21(70)$ & 0.81 \\
\hline CD64 & $59 \pm 26(65)$ & $32 \pm 27(70)$ & $<0.001$ \\
\hline CD11c & $8 \pm 12$ & $53 \pm 27(70)$ & $<0.001$ \\
\hline CD14 & $3 \pm 8(65)$ & $2 \pm 3(70)$ & 0.554 \\
\hline CD56 & $2 \pm 9(64)$ & $21 \pm 32(71)$ & $<0.001$ \\
\hline MPO & $85 \pm 20(65)$ & $87 \pm 21(71)$ & 0.514 \\
\hline $\mathrm{CD} 2$ & $45 \pm 24(25)$ & $33 \pm 34$ & 0.521 \\
\hline
\end{tabular}

APL, acute promyelocytic leukaemia; APTT, activated partial thromboplastin time; PR, prothrombin ratio; SD, standard deviation.

Acknowledgements: The initial work to establish PML IF at LabPlus was reported at the Annual Scientific Meeting of Haematology Society of Australia and New Zealand in Hobart (Tasmania) in 1999 in an oral presentation authored by MLK-Z, MP, PJB and Jane Nelson (JN) entitled 'The PML protein localisation assay as a rapid diagnostic test for acute promyelocytic leukaemia'. MLK-Z developed the assay in our institution under guidance from MP, JN and PJB. Kate Marson and Michelle Charles performed many PML IF tests over the study period.

Conflicts of interest and sources of funding: The work was supported by funding from the School of Medicine Foundation contributed by donations from Anne, Victoria and David Norman. The authors state that there are no conflicts of interest to disclose.

\section{APPENDIX A. SUPPLEMENTARY DATA}

Supplementary data to this article can be found online at https://doi.org/10.1016/j.pathol.2019.01.003.

Address for correspondence: Dr Maggie Kalev-Zylinska, Department of Molecular Medicine and Pathology, University of Auckland, Private Bag 92019, Grafton, Auckland, New Zealand. E-mail: m.kalev@auckland.ac.nz

\section{References}

1. Wang P, Benhenda $\mathrm{S}, \mathrm{Wu} \mathrm{H}$, et al. RING tetramerization is required for nuclear body biogenesis and PML sumoylation. Nat Commun 2018; 9 : 1277.

2. Lallemand-Breitenbach V, de The H. PML nuclear bodies: from architecture to function. Curr Opin Cell Biol 2018; 52: 154-61.

3. Piazza F, Gurrieri C, Pandolfi PP. The theory of APL. Oncogene 2001; 20: $7216-22$.

4. Kayser S, Schlenk RF, Platzbecker U. Management of patients with acute promyelocytic leukemia. Leukemia 2018; 32: 1277-94.

5. Mantha S, Tallman MS, Devlin SM, et al. Predictive factors of fatal bleeding in acute promyelocytic leukemia. Thromb Res 2018; 164 (Suppl 1): S98-102.
6. Swerdlow SH, Campo E, Harris NL, et al., editors. WHO Classification of Tumours of Haematopoietic and Lymphoid Tissues. Lyon: International Agency for Research on Cancer, 2017.

7. Nomdedeu J, Bussaglia E, Villamor N, et al. Immunophenotype of acute myeloid leukemia with NPM mutations: prognostic impact of the leukemic compartment size. Leuk Res 2011; 35: 163-8.

8. Dimov ND, Medeiros LJ, Kantarjian HM, et al. Rapid and reliable confirmation of acute promyelocytic leukemia by immunofluorescence staining with an antipromyelocytic leukemia antibody: the MD Anderson cancer Center experience of 349 patients. Cancer 2010; 116: 369-76.

9. Grimwade D, Howe K, Langabeer S, et al. Establishing the presence of the $\mathrm{t}(15 ; 17)$ in suspected acute promyelocytic leukaemia: cytogenetic, molecular and PML immunofluorescence assessment of patients entered into the MRC ATRA trial. MRC adult leukaemia working party. $\mathrm{Br} J$ Haematol 1996; 94: 557-73.

10. Dyck JA, Warrell Jr RP, Evans RM, et al. Rapid diagnosis of acute promyelocytic leukemia by immunohistochemical localization of PML/ RAR-alpha protein. Blood 1995; 86: 862-7.

11. Falini B, Flenghi L, Fagioli M, et al. Immunocytochemical diagnosis of acute promyelocytic leukemia (M3) with the monoclonal antibody PGM3 (anti-PML). Blood 1997; 90: 4046-53.

12. Voisset E, Moravcsik E, Stratford EW, et al. Pml nuclear body disruption cooperates in APL pathogenesis and impairs DNA damage repair pathways in mice. Blood 2018; 131: 636-48.

13. Adams J, Nassiri M. Acute promyelocytic leukemia: a review and discussion of variant translocations. Arch Pathol Lab Med 2015; 139 $1308-13$.

14. Cullen MJ, Richards SJ, O'Connor SJ, et al. Rapid diagnosis of acute promyelocytic leukemia (PML): applicability of flow cytometry and PML protein immunofluorescence. Cancer Genet Cytogenet 2004: 148: 176-7.

15. Villamor N, Costa D, Aymerich M, et al. Rapid diagnosis of acute promyelocytic leukemia by analyzing the immunocytochemical pattern of the PML protein with the monoclonal antibody PG-M3. Am J Clin Pathol 2000; 114: 786-92.

16. Campbell LJ, Oei P, Brookwell R, et al. FISH detection of PML-RARA fusion in ins $(15 ; 17)$ acute promyelocytic leukaemia depends on probe size. Biomed Res Int 2013; 2013: 164501.

17. Arber DA, Borowitz MJ, Cessna M, et al. Initial diagnostic workup of acute leukemia: guideline from the College of American pathologists and the American Society of hematology. Arch Pathol Lab Med 2017 141: $1342-93$.

18. Sanz MA, Grimwade D, Tallman MS, et al. Management of acute promyelocytic leukemia: recommendations from an expert panel on behalf of the European LeukemiaNet. Blood 2009; 113: 1875-91.

19. Pagnano KB, Rego EM, Rohr S, et al. Guidelines on the diagnosis and treatment for acute promyelocytic leukemia: Associacao Brasileira de Hematologia, Hemoterapia e Terapia Cellular Guidelines Project: 
Associacao Medica Brasileira - 2013. Rev Bras Hematol Hemoter 2014 36: $71-92$.

20. O'Connor SJ, Forsyth PD, Dalal S, et al. The rapid diagnosis of acute promyelocytic leukaemia using PML (5E10) monoclonal antibody. $\mathrm{Br} J$ Haematol 1997; 99: 597-604.

21. Gupta SK, Sazawal S, Mahapatra M, et al. Evaluation of PG-M3 antibody in the diagnosis of acute promyelocytic leukaemia. Eur J Clin Invest 2010; 40: 960-2.

22. Gomis F, Sanz J, Sempere A, et al. Immunofluorescent analysis with the antiPML monoclonal antibody PG-M3 for rapid and accurate genetic diagnosis of acute promyelocytic leukemia. Ann Hematol 2004; 83: 687-90.

23. Cicconi L, Lo-Coco F. Current management of newly diagnosed acute promyelocytic leukemia. Ann Oncol 2016; 27: 1474-81.

24. Seftel MD, Barnett MJ, Couban S, et al. A Canadian consensus on the management of newly diagnosed and relapsed acute promyelocytic leukemia in adults. Curr Oncol 2014; 21: 234-50.

25. Altman JK, Rademaker A, Cull E, et al. Administration of ATRA to newly diagnosed patients with acute promyelocytic leukemia is delayed contributing to early hemorrhagic death. Leuk Res 2013; 37: 1004-9.

26. Falini B, Martelli MP, Tiacci E, et al. Immunohistochemical surrogates for genetic alterations of CCDN1, PML, ALK, and NPM1 genes in lymphomas and acute myeloid leukemia. Best Pract Res Clin Haematol 2010; 23: 417-31.

27. Alayed KM, Medeiros LJ, Phan D, et al. Immunostaining for rapic diagnosis of acute promyelocytic leukemia with the tetramethylrhodamine-5-isothiocyanate-conjugated anti-promyelocytic leukemia monoclonal antibody PG-M3. Arch Pathol Lab Med 2013; 137: $1669-73$

28. Rahman K, Gupta R, Singh MK, et al. The triple-negative (CD34-/HLA DR-/CD11b-) profile rapidly and specifically identifies an acute promyelocytic leukemia. Int J Lab Hematol 2018; 40: 144-51.

29. Dong HY, Kung JX, Bhardwaj V, et al. Flow cytometry rapidly identifies all acute promyelocytic leukemias with high specificity independent of underlying cytogenetic abnormalities. Am J Clin Pathol 2011; 135: 76-84.

30. van Luijn MM, Westers TM, Chamuleau ME, et al. Class II-associated invariant chain peptide expression represents a novel parameter for flow cytometric detection of acute promyelocytic leukemia. Am J Pathol 2011; 179: 2157-61.

31. Chen Z, Li Y, Tong Y, et al. Stepwise discriminant function analysis for rapid identification of acute promyelocytic leukemia from acute myeloid leukemia with multiparameter flow cytometry. Int J Hematol 2016; 103 : $306-15$.

32. Grimwade L, Gudgin E, Bloxham D, et al. PML protein analysis using imaging flow cytometry. J Clin Pathol 2011; 64: 447-50.

33. Mirabelli P, Scalia G, Pascariello C, et al. ImageStream promyelocytic leukemia protein immunolocalization: in search of promyelocytic leukemia cells. Cytometry A 2012; 81: 232-7.

34. Dekking $\mathrm{EH}$, van der Velden $\mathrm{VH}$, Varro $\mathrm{R}$, et al. Flow cytometric immunobead assay for fast and easy detection of PML-RARA fusion proteins for the diagnosis of acute promyelocytic leukemia. Leukemia 2012; 26: 1976-85.

35. Kolhe R, Mangaonkar A, Mansour J, et al. Utility and impact of early $\mathrm{t}(15 ; 17)$ identification by fluorescence in situ hybridization (FISH) in clinical decision making for patients in acute promyelocytic leukemia (APL). Int J Lab Hematol 2015; 37: 515-20.

36. Shigeto S, Matsuda K, Yamaguchi A, et al. Rapid diagnosis of acute promyelocytic leukemia with the PML-RARA fusion gene using a combination of droplet-reverse transcription-polymerase chain reaction and instant-quality fluorescence in situ hybridization. Clin Chim Acte 2016; 453: 38-41.

37. Guo B, Cheng W, Xu Y, et al. A simple surface plasmon resonance biosensor for detection of PML/RARalpha based on heterogeneous fusion gene-triggered nonlinear hybridization chain reaction. Sci Rep 2017; 7: 14037 . 